\title{
First-Degree Relatives of Colorectal Cancer Patients Are Likely to Show Advanced Colorectal Neoplasia despite a Negative Fecal Immunochemical Test
}

\author{
Jae Myung Cha Joung II Lee Kwang Ro Joo Hyun Phil Shin Jae Jun Park \\ Jung Won Jeun Jun Uk Lim \\ Department of Internal Medicine, Kyung Hee University Hospital at Gang Dong, Seoul, Republic of Korea
}

\author{
Key Words \\ Colorectal neoplasia • Fecal immunochemical test • \\ First-degree relatives
}

\begin{abstract}
Background/Aims: Fecal immunochemical test (FIT) is an integral component of most colorectal cancer (CRC) screening programs. However, little is known about clinical risk factors associated with advanced colorectal neoplasia (CRN) despite negative FIT results. The aim of this study was to determine the clinical predictors of advanced CRN despite negative FIT results. Methods: We performed FITs for asymptomatic subjects $\geq 50$ years from January 2009 to December 2010. Patients who underwent colonoscopy for a medical check-up, despite a negative FIT result, were included to evaluate the clinical predictors of advanced CRN based on colonoscopy. Results: During the study period, 373 subjects underwent screening colonoscopy despite their negative FIT results. Among those 373 subjects, 356 (95.4\%) did not show any advanced CRNs; however, 17 (4.6\%) subjects showed advanced CRNs on their colonoscopies despite negative FIT results. Being a first-degree relative of a CRC patient was significantly associated with advanced CRNs in univariate analysis ( $p=$ 0.031). According to multivariate logistic regression analysis, being a first-degree relative of a CRC patient was a signifi-
\end{abstract}

cant predictor of advanced CRNs despite negative FIT results (OR 7.33; 95\% Cl, 0.53-35.08; $p=0.013$ ). Conclusion: Firstdegree relatives of CRC patients are likely to show advanced CRNs despite a negative FIT.

Copyright $\odot 2012$ S. Karger AG, Basel

\section{Introduction}

The incidence of colorectal cancer (CRC) in Asian countries, including Korea, has increased markedly in recent years $[1,2]$. This epidemiologic change in Asia indicates that CRC screening, which is shown to reduce the incidence and mortality of CRC, should be conducted [35]. Among the currently recommended CRC screening modalities, only the fecal occult blood test has been evaluated in the context of a randomized clinical trial for reducing CRC mortality [6, 7]. Although fecal immunochemical tests (FITs) are integral components of most CRC screening programs, they have been criticized because of their poor sensitivity and specificity $[8,9]$. In most population-based CRC screening programs, only individuals with a positive FIT undergo subsequent colonoscopy. Therefore, little is known about clinical risk factors associated with advanced colorectal neoplasia (CRN) despite a negative FIT result. 
The purpose of this study was to identify the clinical predictors of an advanced CRN despite a negative FIT result within the context of a population-based CRC screening program.

\section{Subjects and Methods}

\section{Subjects}

We evaluated asymptomatic subjects aged $\geq 50$ years who underwent screening colonoscopy despite a negative FIT as a part of the National Cancer Screening Program (NCSP) at Gang Dong Kyung Hee University Hospital between January 1, 2009, and December 31, 2010. The NCSP of Korea provided a single annual FIT as an initial CRC screening for subjects aged $\geq 50$ years, who were all Medical Aid recipients and National Health Insurance beneficiaries within the lower $50 \%$ income bracket [10]. All participants were notified of their FIT results, and only subjects with a positive FIT result were invited for colonoscopy. Subjects with a negative FIT result were not advised to receive a colonoscopy; however, some participants underwent a screening colonoscopy despite the negative FIT result at their expense because of the poor sensitivity and specificity of FIT. We evaluated the clinical predictors associated with advanced CRN on colonoscopy despite a negative FIT result. Subjects $<50$ years of age, subjects currently hospitalized, those who refused to participate in a routine CRC screening or had symptoms or signs indicating the need for a colonoscopy were excluded from this study. This study was approved by the Institutional Review Board of the Gang Dong Kyung Hee University Hospital (KHNMC IRB 2011-024); informed consent was not required for this retrospective study.

\section{Definition of False-Negative and True-Negative FIT}

The false-negative and true-negative FITs were classified by defining colonoscopy and histological outcome as a gold standard. A false-negative FIT was defined as the presence of an advanced CRN on colonoscopy despite a negative FIT result. A truenegative FIT was defined as absence of an advanced CRN after a negative FIT. As the relevant finding of a screening colonoscopy was an advanced CRN, CRNs without advanced histology were not included in the false-negative group. An advanced CRN was defined as the presence of carcinoma or advanced adenoma (i.e. adenomas with any villous features, high-grade dysplasia or a dysplasia $>10 \mathrm{~mm}$ in size). The clinicopathological characteristics of the false-negative group were compared with those in the truenegative group.

\section{FIT and Colonoscopy}

FITs were processed with the OC-Sensor Diana Micro desktop analyzer (Eiken Chemical Co., Tokyo, Japan). A hemoglobin concentration $\geq 100 \mathrm{ng} / \mathrm{ml}$ was used as the cut-off value for a positive result. For the FIT, all participants were instructed to sample their stool while preventing contact with water or urine; however, restrictions were not made with regard to either diet or use of medication.

Colonoscopies were performed by experienced gastroenterologists using a standard video colonoscope (EC-590ZW; Fujinon Inc., Saitama, Japan). All patients underwent 3 days of dietary re- striction and bowel preparation that included the ingestion of 4 liters of polyethylene glycol solution or $90 \mathrm{ml}$ of sodium phosphate during the $12 \mathrm{~h}$ preceding the colonoscopy. A complete colonoscopy was defined as intubation of the cecum involving identification of the ileocecal valve and appendiceal orifice. All colonoscopic findings, including the size, shape, location and histological features of polyps, were analyzed. Polyp size was assessed by comparison with a specimen taken with an open-biopsy forceps (Olympus FB-28U-1, Amori Olympus Co., Ltd., Amori, Japan), the larger size being used for multiple adenomas. The detected lesions were classified based on the most advanced colorectal lesions on colonoscopy.

\section{Statistical Analyses}

The clinical predictors of an advanced CRN on colonoscopy despite a negative FIT result were the primary outcome. All data are presented as means \pm standard deviations (SDs) for continuous variables and as number and percentage for categorical variables. Continuous variables were compared between the two groups using the Mann-Whitney $U$ test and categorical variables were compared between the two groups using Fisher's exact test. A two-tailed $p$ value $<0.05$ was considered statistically significant. Statistical analysis was performed using the Statistical Package for the Social Sciences (SPSS) version 13.0 for Windows (SPSS, Chicago, Ill., USA).

\section{Results}

During the study period, 373 subjects underwent screening colonoscopy for a medical check-up despite their negative FIT results. The eligible 373 subjects were composed of $150(40.2 \%)$ men and 223 (49.8\%) women; their mean age was $60.4 \pm 7.2$ years. In total, 132 (35.4\%) subjects showed CRNs; and 241 (64.6\%) subjects did not show any CRNs on their colonoscopies. For advanced CRNs, 356 (95.4\%) subjects did not show any advanced CRNs (true-negative FIT results); however, 17 (4.6\%) subjects showed advanced CRNs on their colonoscopies despite negative FIT results (false-negative FIT results).

Table 1 shows the baseline characteristics of the subjects in the true-negative and false-negative groups. Firstdegree relatives of CRC patients more frequently belonged to the false-negative group ( 25.0 vs. $7.3 \%, \mathrm{p}=$ 0.031 ). However, statistically significant differences were not found for other variables between the false-negative and true-negative groups ( $\mathrm{p}>0.1$ for all).

Multivariate logistic regression analysis was conducted to determine the clinical predictors associated with an advanced CRN despite a negative FIT result (table 2). After adjusting for age, gender, body mass index, prior CRC screening and a prior diagnosis of CRN by conditional multivariate analysis, being a first-degree relative of a CRC patient was the only significant predictor of ad- 
Table 1. Baseline characteristics of the subjects in the true-negative and false-negative groups

\begin{tabular}{lccc}
\hline Clinical characteristic & $\begin{array}{l}\text { True-negative group } \\
(\mathrm{n}=356)\end{array}$ & $\begin{array}{l}\text { False-negative group } \\
(\mathrm{n}=17)\end{array}$ & $\mathrm{p}^{2}$ value \\
\hline Age, years & $60.4 \pm 7.2$ & $59.4 \pm 6.2$ & 0.571 \\
Male gender, $\mathrm{n}$ & $141(39.6 \%)$ & $9(52.9 \%)$ & 0.273 \\
Body mass index & $24.2 \pm 6.9$ & $25.1 \pm 2.0$ & 0.680 \\
First-degree relative of CRC patient, $\mathrm{n}$ & $26(7.3 \%)$ & $4(23.5 \%)$ & 0.031 \\
Prior screening, $\mathrm{n}$ & & $1(5.9 \%)$ & 1.000 \\
$\quad$ Screening with barium enema & $32(9.0 \%)$ & 0.396 \\
$\quad$ Screening with colonoscopy & $58(16.3 \%)$ & $1(5.9 \%)$ & 0.329 \\
Prior diagnosis of any CRN, $\mathrm{n}$ & $3.2 \pm 7.9$ & 0.771 \\
Fecal hemoglobin concentration in FIT, ng/ml & $33(9.2 \%)$ & $1(6.3 \%)$ & 0.610 \\
Inadequate bowel preparation, $\mathrm{n}$ & $408.1 \pm 68.8$ & $391.2 \pm 105.2$ & 0.385 \\
Colonoscopy withdrawal time, $\mathrm{s}$ & & \\
\hline
\end{tabular}

\footnotetext{
${ }^{1}$ Includes subjects without advanced CRN on colonoscopy after a negative FIT result.

${ }^{2}$ Includes subjects with advanced CRN on colonoscopy despite a negative FIT result.
}

Table 2. Multivariate analysis of clinical predictors for an advanced CRN despite negative FIT

\begin{tabular}{|c|c|c|c|}
\hline Parameters & & Odds ratio $(95 \% \mathrm{CI})$ & $\mathrm{p}$ value \\
\hline \multirow{2}{*}{ Age group } & $<65$ years & 1 & \\
\hline & $\geq 65$ years & $3.52(0.41-30.16)$ & 0.250 \\
\hline \multirow[t]{2}{*}{ Gender } & male & 1 & \\
\hline & female & $1.63(0.45-5.96)$ & 0.458 \\
\hline \multirow[t]{2}{*}{ Body mass index } & $\geq 25$ & 1 & \\
\hline & $<25$ & $1.23(0.29-5.17)$ & 0.779 \\
\hline \multirow{2}{*}{$\begin{array}{l}\text { First-degree relative } \\
\text { of CRC patient }\end{array}$} & no & 1 & \\
\hline & yes & $7.33(0.53-35.08)$ & 0.013 \\
\hline \multirow{2}{*}{$\begin{array}{l}\text { Prior colorectal } \\
\text { cancer screening }\end{array}$} & yes & 1 & \\
\hline & no & $0.56(0.13-2.36)$ & 0.433 \\
\hline \multirow{2}{*}{$\begin{array}{l}\text { Prior diagnosis of } \\
\text { colorectal neoplasia }\end{array}$} & no & 1 & \\
\hline & yes & - & 0.998 \\
\hline
\end{tabular}

vanced CRN despite a negative FIT result (OR 7.33; 95\% CI $0.53-35.08 ; \mathrm{p}=0.013)$. First-degree relatives of CRC patients were 7.33 times more likely to have an advanced CRN despite a negative FIT result than those with no family history of CRC.

Figure 1 shows the colonoscopic findings of advanced CRNs in the false-negative group. A total of 25 advanced CRNs was detected in 17 patients of the false-negative group. The mean number of adenomas was $2.8 \pm 1.8$ per patient. Early carcinomas were diagnosed in 3 subjects in the false-negative group despite their negative FITs. In the per-advanced CRN analysis, the mean number was

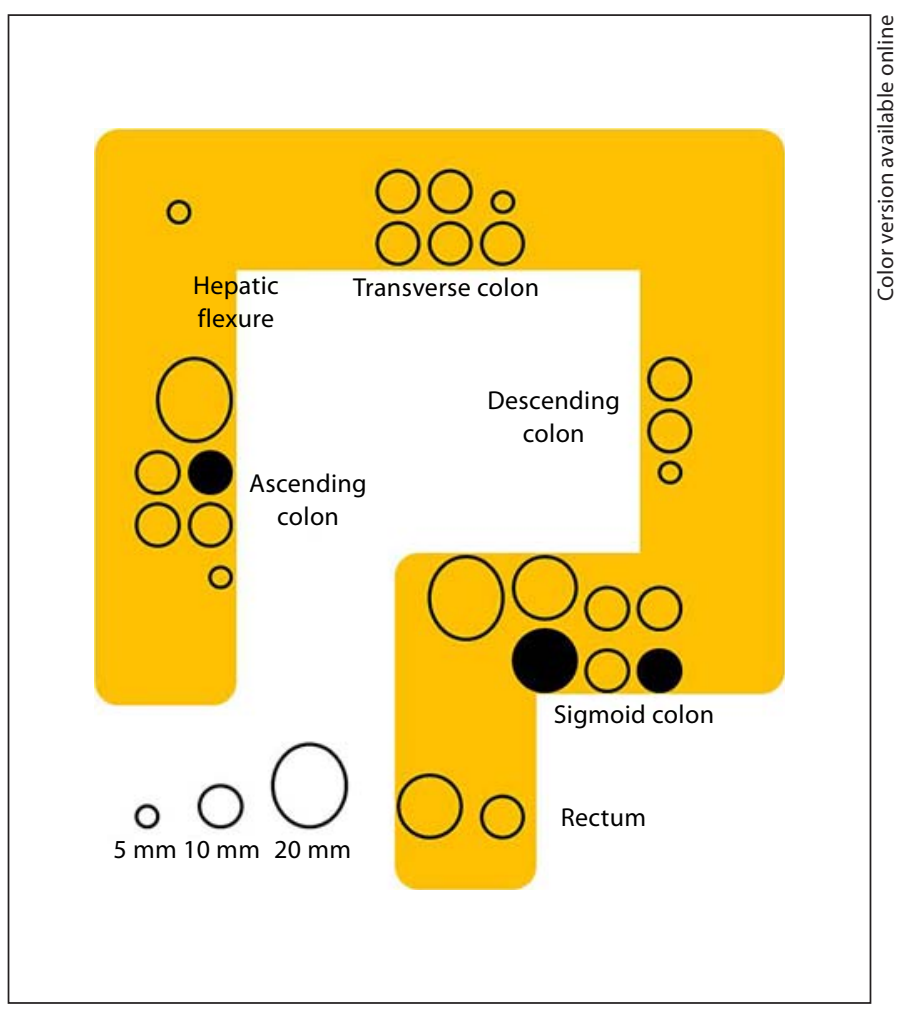

Fig. 1. The colonoscopic findings of 25 advanced CRNs in falsenegative groups. An advanced CRN was expressed as a circle, whose size depends on the size of the CRN and whose location corresponds to the location of the CRN. Moreover, an open circle represents a polypoid configuration whereas a closed circle represents a nonpolypoid configuration. Advanced CRNs were located in the right colon in $52 \%(\mathrm{n}=13)$ and in the left colon in $48 \%$ $(\mathrm{n}=12)$. Most $(88 \%)$ of them displayed polypoid configurations; only $12 \%(n=3)$ of them had nonpolypoid configurations. 
$1.5 \pm 1.2$ and the mean size was $10.6 \pm 3.9 \mathrm{~mm}$. Advanced CRNs were located on the right colon in $52 \%$ ( $n=$ $13)$ and the left colon in $48 \%(n=12)$. As to the shape of advanced CRNs, most (88\%) of them were of polypoid configuration and only $12 \%(\mathrm{n}=3)$ displayed a nonpolypoid configuration.

\section{Discussion}

This is the first study evaluating the clinical predictors associated with an advanced CRN on colonoscopy despite a negative FIT result within the context of a populationbased CRC screening. Our study demonstrated that $35.4 \%$ of the subjects had CRNs and $4.6 \%$ of subjects had advanced CRNs (including 3 cases of early carcinoma) on colonoscopy despite their negative FITs. First-degree relatives of CRC patients more frequently belonged to the false-negative group $(\mathrm{p}=0.031)$. Furthermore, being a first-degree relative of a CRC patient was the only significant predictor of advanced CRNs despite a negative FIT according to multivariate analysis (OR 7.33; 95\% CI $0.53-$ 35.08; $\mathrm{p}=0.013$ ). Because false-negative FIT results may lead to a missed or delayed diagnosis of CRC, identification of clinical predictors associated with a false-negative FIT result may be important in clinical practice. In our study, no different biological characteristics (number, size, location and shape) of advanced CRNs were identified in first-degree relatives of CRC patients; however, a higher trend for an advanced histology was associated with firstdegree relatives of CRC patients (33.3 vs. 9.9\%; $\mathrm{p}=0.069$ ).

Although FIT is a central component of most CRC screening programs [11-15], little is known about the colonoscopic findings and the clinical predictors of advanced CRNs after a negative FIT as screening colonoscopies are not widely practiced for these patients after a negative FIT. In this regard, our study appears to have considerable potential as our data focused on colonoscopic findings in subjects with negative FITs who had been recruited within a population-based CRC screening program. In general, approximately $10-15 \%$ of CRCs occur in first-degree relatives of CRC patients, and these patients have about twice the risk of that of the general population $[16,17]$. Therefore, it is not surprising that first-degree relatives of CRC patients had advanced CRNs despite a negative FIT result in the present study. Firstdegree relatives of CRC patients should be cautiously followed up with repeated FITs or screened with colonoscopy to improve the overall effectiveness of CRC detection. As FIT may result in a considerable number of false-negative cases, the effectiveness of repeated FIT screening should be emphasized in first-degree relatives of CRC patients [18]. Guillem et al. [19] suggested the use of colonoscopy as a routine screening tool in asymptomatic first-degree relatives of CRC patients. In addition, a family-oriented approach may have the potential to capitalize on targeting family clusters by inviting first-degree relatives of CRC patients to take part in a family risk counseling session [20]. The 3 cases of early carcinoma detected in our study despite negative FITs might have been effectively detected via repeated FITs or colonoscopy.

In a population-based screening, the specificity and false-negative rate of an FIT in detecting CRC may be complicated to establish because patients with a negative FIT are not typically considered for colonoscopy. The likelihood of a false-negative FIT result may depend on the biological characteristics of the advanced CRN, i.e. the amount and periodicity of bleeding from advanced CRNs. Other factors, such as storage conditions and lag time before analysis, may also influence the rate of falsenegative FIT results due to hemoglobin degradation in the fecal sample [18]. In the present study, fecal samples were stored at $4^{\circ} \mathrm{C}$ once received by the laboratory, and analyzed usually within $24-48 \mathrm{~h}$. Therefore, false-negative FITs due to storage conditions or lag time before analysis would have been rare in our study.

Our study has three main strengths: First, it is based on a population-based, national CRC screening program and included only asymptomatic participants. Oort et al. [21] showed the performance characteristics of the FIT based on 1,821 referral patients who underwent complete colonoscopy; however, their study population consisted of more individuals with advanced CRNs than in the average screening population. The study population is important as performance characteristics of the FIT using a specific study population may lead to biased results. Second, we constructed a multiple-regression model containing possible confounders of false-negative FIT results. Third, the data collected were of high quality despite its retrospective design, i.e. questionnaires were completed by trained nurses and included questions pertaining to family history of CRC, prior CRC screening and prior diagnosis of colorectal neoplasm.

Some limitations of our study may merit discussion. The most accurate means of estimating the performance characteristics of FITs would be full colonoscopy in all participants who received FITs. However, our study included participants who underwent colonoscopy for a medical check-up despite a negative FIT result, and the 
decision regarding the acceptance of colonoscopy resided solely with the patient. Although this study did not include all patients who had received an FIT, a selection bias is very unlikely as colonoscopy was performed in the frame of a routine medical check-up, and patients with symptoms were excluded during the initial FIT screenings. Furthermore, the possibility of a selection bias according to a family history of CRC patients may also be minimal as only $8.0 \%$ of the participants had a family history in this study, which is lower than the prevalence of a family history of CRC in the general population [22]. However, our findings cannot be generalized as we only enrolled subjects who visited our hospital for FIT under the NCSP and they were largely composed of local, urban adults $\geq 50$ years of age.
In conclusion, being a first-degree relative of a CRC patient was the significant clinical predictor of advanced CRNs despite a negative FIT. Therefore, a first-degree relative of a CRC patient should be closely followed up. However, prospective validation studies on this issue are warranted in a variety of population-based CRC screening programs.

\section{Disclosure Statement}

None of the authors declared any conflicts of interest or disclosed any financial relationship relevant to this publication.

\section{References}

1 Choi KS, Jun JK, Lee HY, Hahm MI, Oh JH, Park EC: Increasing uptake of colorectal cancer screening in Korea: a populationbased study. BMC Public Health 2010;10: 265.

-2 Shim JI, Kim Y, Han MA, Lee HY, Choi KS, Jun JK, Park EC: Results of colorectal cancer screening of the national cancer screening program in Korea, 2008. Cancer Res Treat 2010;42:191-198.

- 3 Winawer SJ, Flehinger BJ, Schottenfeld D, Miller DG: Screening of colorectal cancer with fecal occult blood testing and sigmoidoscopy. J Natl Cancer Inst 1993;85:1311 1318.

4 Mandel JS, Church TR, Bond JH, Ederer F, Geisser MS, Mongin SJ, Snover DC, Schuman LM: The effect of fecal occult-blood screening on the incidence of colorectal cancer. $\mathrm{N}$ Engl J Med 2000;343:1603-1607.

5 Atkin WS, Cuzick J, Northover JM, Whynes DK: Prevention of colorectal cancer by onceonly sigmoidoscopy. Lancet 1993;341:736740 .

6 Hardcastle JD, Chamberlain JO, Robinson MH, Moss SM, Amar SS, Balfour TW, James PD, Mangham CM: Randomised controlled trial of faecal-occult-blood screening for colorectal cancer. Lancet 1996;348:1472 1477.

7 Kronborg O, Fenger C, Olsen J, Jørgensen OD, Søndergaard O: Randomised study of screening for colorectal cancer with faecaloccult-blood test. Lancet 1996;348:14671471.
8 Liberman D, Sleisenger MH: It is time to recommend screening for colorectal cancer? Lancet 1996;348:1463-1464.

-9 Lang CA, Ransohoff DF: Fecal occult blood screening for colorectal cancer. Is mortality reduced by chance selection for screening colonoscopy? JAMA 1994;271:1011-1013.

10 Yoo KY: Cancer control activities in the republic of Korea. Jpn J Clin Oncol 2008;38: 327-333.

11 Toribara NW, Sleisenger MH: Screening for colon cancer. N Engl J Med 1995;332:861867.

$\checkmark 12$ Kronborg O, Fenger C, Olsen J, Jørgensen OD, Søndergaard O: Randomised study of screening for colorectal cancer with faecaloccult-blood test. Lancet 1996;348:14671477.

13 Liberman DA: Colon cancer screening. Obstacles to effectiveness. Cancer 1992;70: 1259-1265.

14 Mandel JS: Colorectal cancer screening. Cancer metastasis Rev 1997;16:263-279.

15 Anonymous: Suggested technique for fecal occult blood testing and interpretation in colorectal cancer screening. American College of Physicians. Ann Intern Med 1997;126: 808-810.

16 Johns LE, Houlston RS: A systematic review and meta-analysis of familial cancer risk. Am J Gastroenterol 2001;96:2992-3003.
17 Butterworth AS, Higgins JPT, Pharoah P: Relative and absolute risk of colorectal cancer for individuals with a family history: a meta-analysis. Eur J Cancer 2006;42:216227.

18 van Rossum LG, van Rijn AF, van Oijen MG Fockens P, Laheij RJ, Verbeek AL, Jansen JB, Dekker E: False negative fecal occult blood tests due to delayed sample return in colorectal cancer screening. Int J Cancer 2009;125: 746-750.

19 Guillem JG, Forde KA, Treat MR, Neugut AI, O'Toole KM, Diamond BE: Colonoscopic screening for neoplasms in asymptomatic first-degree relatives of colon cancer patients. A controlled, prospective study. Dis Colon Rectum 1992;35:523-529.

20 Glanz K, Steffen AD, Taglialatela LA: Effects of colon cancer risk counseling for first-degree relatives. Cancer Epidemiol Biomarkers Prev 2007; 16:1485-1491.

21 Oort FA, Terhaar Sive Droste JS, Van Der Hulst RW, Van Heukelem HA, Loffeld RJ, Wesdorp IC, Van Wanrooij RL, De Baaij L, Mutsaers ER, van der Reijt S, Coupe VM, Berkhof J, Bouman AA, Meijer GA, Mulder CJ: Colonoscopy-controlled intra-individual comparisons to screen relevant neoplasia: faecal immunochemical test vs. guaiacbased faecal occult blood test. Aliment Pharmacol Ther 2010;31:432-439.

-22 Mitchell RJ, Campbell H, Farrington SM, Brewster DH, Porteous ME, Dunlop MG: Prevalence of family history of colorectal cancer in the general population. Br J Surg 2005;92:1161-1164. 